\title{
Role of liver ICAM-1 in metastasis (Review)
}

\author{
AITOR BENEDICTO, IRENE ROMAYOR and BEATRIZ ARTETA
}

\author{
Department of Cell Biology and Histology, School of Medicine and Nursing, \\ University of The Basque Country, UPV/EHU, Leioa, E-48940 Vizcaya, Spain
}

Received January 17, 2017; Accepted July 7, 2017

DOI: $10.3892 / 01.2017 .6700$

\begin{abstract}
Intercellular adhesion molecule (ICAM)-1, is a transmembrane glycoprotein of the immunoglobulin (Ig)-like superfamily, consisting of five extracellular Ig-like domains, a transmembrane domain and a short cytoplasmic tail. ICAM-1 is expressed in various cell types, including endothelial cells and leukocytes, and is involved in several physiological processes. Furthermore, it has additionally been reported to be expressed in various cancer cells, including melanoma, colorectal cancer and lymphoma. The majority of studies to date have focused on the expression of the ICAM-1 on the surface of tumor cells, without research into ICAM-1 expression at sites of metastasis. Cancer cells frequently metastasize to the liver, due to its unique physiology and specialized liver sinusoid capillary network. Liver sinusoidal endothelial cells constitutively express ICAM-1, which is upregulated under inflammatory conditions. Furthermore, liver ICAM-1 may be important during the development of liver metastasis. Therefore, it is necessary to improve the understanding of the mechanisms mediated by this adhesion molecule in order to develop host-directed anticancer therapies.
\end{abstract}

\section{Contents}

1. Introduction

2. ICAM-1 in healthy liver

3. ICAM-1 in liver metastasis

4. Conclusions

\section{Introduction}

Currently, cancer is one of most common causes of mortality worldwide. Colorectal cancer (CRC) represents one of the most

Correspondence to: Professor Beatriz Arteta, Department of Cell Biology and Histology, School of Medicine and Nursing, University of The Basque Country, UPV/EHU, Barrio Sarriena, Leioa, E-48940 Vizcaya, Spain

E-mail: beatriz.arteta@ehu.eus

Key words: liver metastasis, tumor microenvironment, adhesion molecules, intercellular adhesion molecule-1, invasion, immune response, angiogenesis frequently diagnosed cancer types and is the fourth leading cause of cancer-related death (1). The spreading of cells from the primary lesion to a secondary organ and the subsequent development of distant metastases is a key factor that limits patient survival rate. This remains one of the most complex issues faced in medicine (2).

The liver is the main target organ for metastatic CRC cells and the second most commonly invaded organ, after the lymph nodes (3). In fact, 15-25\% of CRC patients present with synchronous hepatic metastases at the time of diagnosis, and a further $30 \%$ will later develop liver metastasis $(4,5)$. The complex network of vessels and microcapillaries of the hepatic microcirculation makes the liver a target for circulating cells (6). Indeed, cancer cells released from a primary lesion follow a natural blood flow directly to the liver, through the specialized microvessel network known as the liver sinusoids. Gastric cancers also commonly metastasize to the liver (7). Circulating cells from other primary malignancies, such as melanoma, breast or neuroendocrine tumors (8-10), also adhere, establish and develop in the liver, giving rise to metastases, although this is less common.

Metastatic progression is a highly complex and coordinated cascade of events that is influenced by a wide variety of mediators $(11,12)$. Among the key factors that participate in this process, adhesion molecules expressed on cancer cells and cells of the target organ have a crucial role $(13,14)$. Adhesion molecules generate the initial cell-cell contacts that lead to cancer cell extravasation and organ colonization. Additionally, these proteins may also act as signaling molecules to modulate the local microenvironment, creating a pro-metastatic environment, and trigger an angiogenic and desmoplastic response via a complex reciprocal dialogue between the tumor cells and the cells of the colonized organ $(15,16)$. In addition to the tissue cells, immune populations recruited from the circulation during metastasis formation are also involved in generating a favorable environment for metastatic growth $(17,18)$. Therefore, determining the role of adhesion molecules during the different stages of this process remains a major goal for our understanding of the metastatic cascade. This, in turn, will facilitate new opportunities for therapeutic intervention.

To date, the research effort undertaken to investigate the function of adhesion proteins expressed on the surface of tumor cells and their implications in organ colonization has increased our knowledge about the signaling pathways that operate in tumor cells. The 'seed and soil' theory (19) postulates that host organ-specific adhesion molecules are 
required for the switch towards invasion and disease progression (20,21). Several adhesion molecules, such as E-selectin, vascular cellular adhesion molecule (VCAM)-1 and ICAM-1, exhibit increased expression in the liver during metastatic invasion (22). Among them, ICAM-1 mediates several stages of the metastatic cascade, including the adhesion of tumor cells to the endothelial wall (23-25), endothelial cell activation of pro-metastatic signaling pathways (26-28), tumor cell extravasation $(23,29)$, the recruitment of immune cell populations $(28,30,31)$, the pro-angiogenic response (32) and the transdifferentiation of stellate cells during the desmoplastic response $(33,34)$. This review will focus on the role of ICAM-1 during the different events of the metastatic cascade that drives colonization of the liver by circulating tumor cells, and how it modulates the liver microenvironment to facilitate metastasis.

\section{ICAM-1 in healthy liver}

ICAM-1 is a transmembrane glycoprotein of the immunoglobulin (Ig)-like superfamily, consisting of five extracellular Ig-like domains, a transmembrane domain and a short cytoplasmic tail (35). This transmembrane domain is essential for cell-cell adhesion and cell-extracellular matrix (ECM) interaction $(36,37)$. In the liver, ICAM-1 is expressed constitutively in liver sinusoidal endothelial cells (LSECs), hepatocytes, Kupffer cells (KCs) and hepatic stellate cells (HSCs) $(33,38,39)$, and is and further upregulated by inflammatory activation, such as stimulation by TNF- $\alpha$, IL- $1 \beta$ or IFN- $\gamma$ (40). As in other organs, inflammation is accompanied by the recruitment of multiple immune cell populations, such as neutrophils, lymphocytes and monocytes. Leukocytes invade the tissue after crossing the liver endothelium via interaction between endothelial ICAM-1 $(41,42)$ and its main counter-receptor, lymphocyte function-associated antigen (LFA)-1, on lymphocytes.

\section{ICAM-1 in liver metastasis}

ICAM-1 appears to have a major role during the initiation of the metastatic cascade driving tumor progression. The expression of ICAM-1 protein by liver parenchymal and non-parenchymal cells enhances its potential to facilitate disease progression (Fig. 1). In fact, ligand binding to ICAM-1 in LSECs and $\mathrm{KCs}$, and ICAM-1 overexpression in HSCs and hepatocytes $(26,37,43,44)$ contribute to the activation of multiple signaling pathways with key roles in different stages of metastatic progression. Furthermore, the soluble form, sICAM-1, enhances the pro-metastatic phenotype and the pro-inflammatory and pro-tumoral signaling (45). sICAM-1 has been demonstrated to be elevated in the serum of patients with liver metastasis from lung or gastric cancer $(46,47)$, and has been identified as a marker for metastatic stage, disease recurrence and prognosis in non-Hodgkin's lymphoma, hepatocellular carcinoma (HCC), lung cancer, and other cancer types (48-50).

Tumor cell adhesion: Lending tumor cells a hand. The adhesion to LSECs and the ulterior extravasation of the malignant tumor cells across this endothelial line into the liver represents the first step in liver metastatic colonization (20). Invading cells initially require an anchor to adhere to and help them escape from the blood stream (Fig. 2). At this time, endothelial ICAM-1 is involved in tumor cell adhesion to the endothelium; this is a particularly important phenomenon considering that tumor adherence to vessel walls is a common feature across numerous types of cancer (51). Ghislin et al reported that ICAM-1 expressed on the surface of endothelial cells is crucial for the adhesion of melanoma cells to the endothelial monolayer in vitro. Under these conditions, ICAM-1 expression is increased after tumor stimulation, in parallel with an increase in tumor cell adhesion, and this effect can be abrogated by the treatment of the endothelial cells with specific anti-ICAM-1 antibodies (29). These results are consistent with another report that showed that tumor cell interaction with endothelial cells increases ICAM-1 expression on the endothelial cell surface (52). Furthermore, expression of ICAM-1 was shown to be correlated with the production of pro-tumoral cytokines, such as IL-8 and IL-6. IL-8 and IL-6 facilitate tumor cell attachment to endothelial cells and enhance vascular permeability, as observed in models of brain and lung metastasis $(53,54)$. Additionally, a previous study used atomic-force microscopy to show that endothelial ICAM-1 mediates the adhesion of different invasive bladder cancer cells to endothelial cells (24). In line with these results, a reduction in ICAM-1 expression via siRNAs or by using ICAM-1-blocking antibodies significantly decreased the adhesion of fibrosarcoma cells to ECV304 human endothelial cells in vitro (55), and of C26 CRC cells to LSECs in vivo (56). Furthermore, the adhesion of different tumor cells may be reduced by blocking the expression of ICAM-1 in the endothelium of either brain or lung, leading to the abrogation of metastasis to these organs, which further confirms the role of the ICAM-1 in tumor cells $(53,54)$. Moreover, ICAM-1 can cooperate with other adhesion molecules, such as VCAM-1, in the adhesion of malignant cells. ICAM-1 and VCAM-1 are both upregulated in a TNF- $\alpha$-dependent manner (TNF- $\alpha$ being predominantly produced by macrophages) (57). Notably, LSECs lack the ability to express selectins, which are basally expressed and inducible in the endothelium of the portal tract and central vein (58). Likewise, this may have implications for the pathophysiology of the liver and may affect the normal distribution of tumor cell adhesion during the first steps of infiltration. In parallel with the upregulation of E-selectin, ICAM-1 and VCAM-1 in the liver, the production of pro-inflammatory cytokines is also increased in HCC (59). Among them, IL- 6 is implicated in the attraction of tumor cells, initiating a positive feedback response, and thus, not only promotes the progression of the metastasis, but also increases the risk of recurrence. Furthermore, the adhesion of CRC cells to KCs through carcinoembryonic antigen (CEA), increases the release of cytokines from KCs, including IL-1, TNF and IL-6, which induces ICAM-1 upregulation in ECV304 human endothelial cells and increases tumor cell adhesion to these cells in vitro (40).

All these results support that endothelial ICAM-1 has a crucial role in the adhesion of cancer cells to the endothelium in target organs and, thus, in the progression of cancer. Interestingly, ICAM-1 expression has been recently linked to a unique mechanism of leukocyte adhesion that specifically occurs in the liver (60), in contrast to the classic rolling-adhesion-diapedesis mechanisms used by leukocytes in many other organs. It 


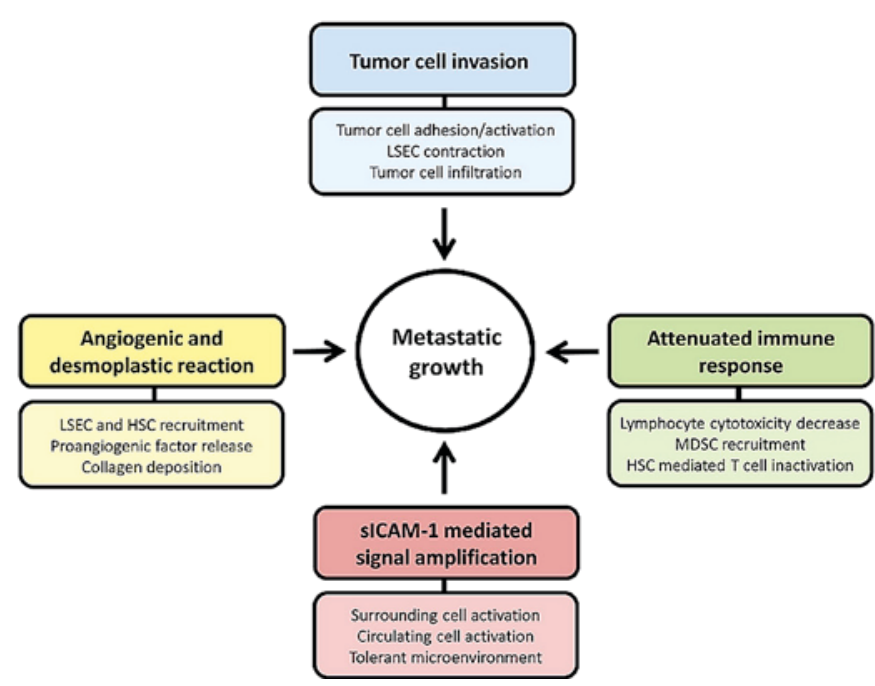

Figure 1. Mechanisms involving ICAM-1 during liver metastasis. The ligation and secretion of sICAM-1 burst metastatic progression through different mechanisms, such as tumor cell invasion, attenuated immune response, promoting angiogenic and desmoplastic response and expanding ICAM-1 mediated effects through its soluble form. ICAM-1, intercellular adhesion molecule-1.

is tempting to hypothesize that leukocyte adhesion molecules expressed on tumor cells and ICAM-1 in LSECs may also been involved in the events leading to cancer cell colonization of the liver. If so, ICAM-1 expressed on LSECs could be crucial for the development of liver metastasis, and a potential candidate for the development of new and improved targeted therapies.

The majority of the studies conducted to investigate the role of ICAM-1 in liver metastasis have mainly focused on its expression on cancer cells. Studies involving surface ligands on the tumor, such as MUC- 1 and the $\beta 2$ integrin of the LFA-1 receptor, known to bind to ICAM-1 specifically (61), have provided evidence of the role of this Ig superfamily molecule in metastasis when expressed on the host cells. The expression of different ICAM-1 ligands has been detected in a wide variety of cancer types. In fact, $\beta 2$ integrin is expressed in melanoma, lymphoma, myeloma, gastrointestinal carcinomas, and was recently reported in breast cancer (29,62-65). Another ICAM-1 ligand, MUC-1, is expressed on breast cancer cells (23), as well as ovarian, prostate, gastric and pancreatic cancer cells and liver metastases (66). The hyaluronan receptor CD44 and its isoforms, which can be used alongside others markers for cancer stem cell identification (67), are expressed on breast, colorectal and pancreatic cancers cells (68-70). The increasing evidence of tumor cell expression of ICAM-1 ligands, and of the interaction between tumor cells and host ICAM-1, demonstrate that the significance of host cell ICAM-1 expression during the course of liver metastasis, and knowledge of role of ICAM-1 expression, may increase in the coming years. Consistently with this idea, the adhesion of LFA-1-expressing C26 CRC cells to LSECs was shown to be reduced by blocking ICAM-1 with specific antibodies $(27,32)$. The same result was observed when the expression of $\beta 2$ integrin in $\mathrm{C} 26$ cells was reduced via siRNA (Benedicto et al, submitted). Additionally, the expression of other ICAM-1 ligands, such as MUC-1, in breast cells was shown to mediate the adhesion of the malignant cells to HUVECs (25).
Interestingly, the vessels located at the invasive front of CRC tumors exhibit higher expression of ICAM-1. These vessels are considered to be a gateway for the entry of inflammatory cells into cancer tissue (71). Along with endothelial cells, ICAM-1 expression by other resident liver cells has been also studied. The resident macrophages of the liver, KCs, also express ICAM-1 (72). Some reports in other cancer types have suggested that the adhesion of macrophages to tumor cells promotes metastasis (73). It is tempting to hypothesize that expression of specific ligands may mediate the adhesion of tumor cells to macrophages expressing ICAM-1, further increasing liver colonization by circulating cancer cells. Additionally, tumor-activated $\mathrm{KCs}$, and HSCs, which also express ICAM-1 when activated by inflammatory factors (74), further stimulate the expression of ICAM-1 on the surface of endothelial cells, increasing vascular permeability, which is also associated with an increase in tumor cell invasion into a target organ (75).

Tumor cell extravasation: Opening the liver's doors to invading cells. Endothelial cells are the first barrier that tumor cells encounter when invading the liver (51), since they act as gatekeepers, allowing the infiltration of cells only when required. Once adhered to LSECs, cancer cells must pass across the endothelium through a process called diapedesis in order to extravasate $(76,77)$. The contraction of endothelial cells through actin cytoskeletal reorganization is essential for the infiltration of invading cells $(78,79)$. The remodeling of the cytoskeleton is also observed after the binding of endothelial ICAM-1 to leukocyte LFA-1 during transmigration of immune cells, including leukocytes, lymphocytes and neutrophils $(44,80,81)$. In this scenario, ICAM-1 expression has also been reported to be correlated with efficient transmigration across the endothelial cell layer and the subsequent extravasation of tumor cells, which in turn, facilitates the colonization of the organ $(29,82,83)$. While elucidating the mechanisms of the metastatic process, it has been proposed that tumor cells mimic the route of immune cells, by binding to endothelial ICAM-1 and forcing their way through the endothelial wall fenestrations to invade the tissue parenchyma. In contrast to other organs, where leukocyte recruitment depends on the expression of selectins on post-capillary venules, leukocyte adhesion to the hepatic sinusoidal endothelium depends on ICAM-1 (84). This is consistent with the lack of selectins observed in LSECs and the limited expression of ICAM-1 in portal tracts under basal conditions. In a previous study, neutralization of endothelial ICAM-1 and VCAM-1 by specific antibodies in a leukemia model was reported to abrogate cancer cell diapedesis across human coronary artery endothelial cell wall, which further supports the idea that endothelial ICAM-1 is involved in cancer cell invasion to the liver through regulation of transmigration and extravasation (85). Furthermore, in a melanoma model, Ghislin et al showed that blocking the LFA-1/ICAM-1 interaction using specific antibodies either to ICAM-1 on endothelial cells, or to the CD18 subunit of the LFA-1 integrin in tumor cells, reduced transendothelial migration (29). Moreover, transendothelial migration of breast carcinoma cells is promoted by the interaction between tumor MUC-1 and ICAM-1 expressed on the endothelial surface (23). Together these observations suggest that ICAM-1 has an 


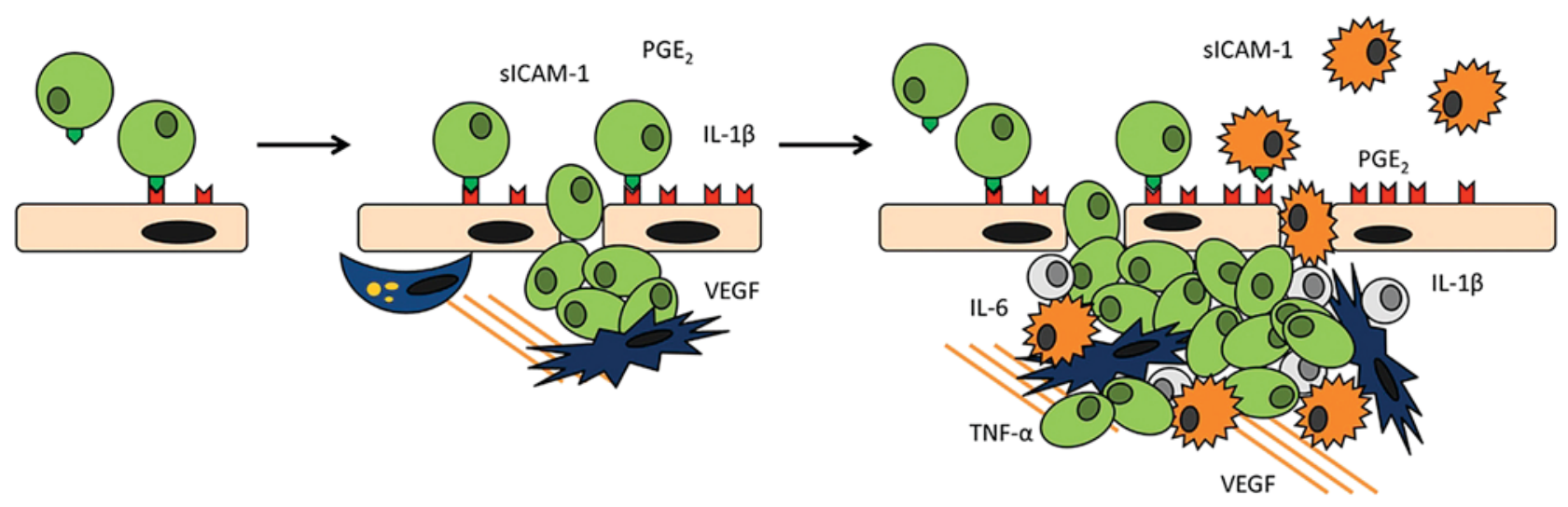

ICAM-1 ligation.

ICAM-1 overexpression in LSEC. IL- $1 \beta$ and SICAM- 1 secretion by LSEC. Tumor $\mathrm{PGE}_{2}$ and VEGF release.

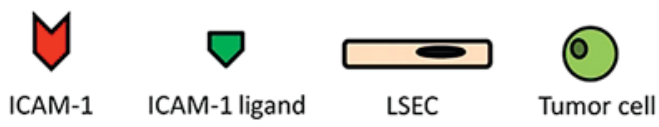

\begin{abstract}
LSEC cytoskeletal reorganization. Tumor cell extravasation and activation. LSEC and HSC recruitment.
\end{abstract}

MDSC recruitment and infiltration. Lymphocyte cytotoxicity decrease. Cytokines and growth factor release.

Figure 2. ICAM-1 mediated protumoral pathways. ICAM-1 ligation in LSECs triggers the secretion of sICAM-1 and IL-1 $\beta$ concomitant to the upregulation of ManR causing a decrease in the cytotoxic potential of LSLs. Furthermore, ICAM-1 activated tumor cells secrete VEGF and PGE 2 facilitating the recruitment of MDSCs, LSECs and HSCs leading to angiogenesis. HSCs block lymphocyte mediated tumor clearance by interacting with lymphocyte ICAM-1. Moreover, MDSCs infiltrate the liver through a process involving upregulation of ICAM-1 expression in LSECs. Consequently, the antitumor response of infiltrating and resident lymphocytes is impeded. Additionally, ICAM-1 ligation in stromal cells drives to TNF- $\alpha$, and IL-6 release, amplifying the effect on tumor progression. ICAM-1, intercellular adhesion molecule-1; LSECs, liver sinusoidal endothelial cells; VEGF, vascular endothelial growth factor; MDSCs, myeloid-derived suppressor cells; HSCs, liver sinusoidal endothelial cells.

important role in organ invasion by circulating tumor cells, although the downstream mechanisms remain unknown. As for leukocytes, during sterile inflammation (86), tumor cells may utilize ICAM-1 to infiltrate the liver parenchyma in order to colonize the organ. Recent studies from our group revealed that there is a direct link between the tumor LFA-1/endothelial ICAM-1 interaction and the transmigration of CRC cells through the endothelial cell lining of the liver sinusoids, since a decrease in the number of transmigrated cells was observed when either the $\beta 2$ integrin of tumor cells (Benedicto et al, submitted) or the ICAM-1 of endothelial cells are silenced. The perturbation of this interaction reduced tumor cell migration through an endothelial monolayer in vitro, and demonstrates the complexity of metastasis and the signaling mechanisms involved in this process in the liver.

Tumor promotion: Laying the ground for metastatic development. Due to its physiological location, the liver has a chronic inflammatory microenvironment, where the immune system must continuously fight against pathogens from within the intestinal tract. In fact, the cells residing in the liver, namely hepatocytes, KCs, LSECs and HSCs, are involved in the inflammatory cascade (87) and maintaining homeostasis within the organ. However, this inflammatory status is a double-edged sword, since cancer development has been shown to be profoundly enhanced by pro-inflammatory signals (88). The creation of a pro-tumor microenvironment is required for cancer cell proliferation and for tumors to evade immune surveillance, leading to a decreased immune recognition and/or elimination of reactive immune cells. Modulation of the target organ pro-metastatic response is initiated by interaction of tumor cells with endothelial cells, leading to the activation of these vascular cells and secretion of pro-inflammatory cytokines and growth factors (89). The role of ICAM-1 in the creation of such an environment was reported by Arteta et al (27). The authors reported that ICAM-1 binding with tumor LFA-1 triggered the production of IL-1 $\beta$ by LSECs, which in turn promoted mannose receptor (ManR)-mediated endocytosis. The increase in the activity and expression of ManR was correlated with a decreased cytotoxic effect of liver sinusoidal lymphocytes on the C26 murine CRC cell line (27). This defective immune response facilitates the establishment and development of metastasis, as one of the hallmarks of tumor progression (90).

During liver metastasis, liver tissue is replaced by the continuously growing tumor mass, along with the activated resident liver cells, creating a dynamic network that has been termed a 'wound that never heals' (91). During this 'healing' process after liver damage, liver myofibroblast-like cells, also known as HSCs, have a key role (92). This contractile cell population are particularly important in relation to cancer, since cancer-associated fibroblasts (CAFs) have also been identified as a key cell type that favor tumor growth and development (93). Intriguingly, the expression of ICAM-1 has been shown to be induced in activated HSCs (94), in a mechanism mediated by cytokines and growth factors, such as TNF- $\alpha$ (74), as well as by cell-cell contacts. It is interesting to note that the stellate cells present in other organs with tumors that mainly metastasize to the liver, such as pancreatic carcinomas, also express ICAM-1 when activated (95). Furthermore, the increased expression of ICAM-1 on the surface of HSCs, activated by infiltrating lymphocytes, leads to tumor growth 
and immune evasion (33). On the one hand, HSCs are able to promote tumor proliferation via the secretion of a wide array of soluble mediators (96), which potentially modulate the tumor microenvironment. Additionally, HSC activation by melanoma-derived soluble factors results in the recruitment of LSECs, promoting the development of new vessels (97). On the other hand, activated HSCs have the ability to phagocytose lymphocytes during liver inflammation (98), to abrogate CD8 ${ }^{+}$ $\mathrm{T}$ cell-mediated immunity (99), and to enhance the recruitment of immunosuppressive cells (100), thus impeding the appropriate anti-tumor function in an ICAM-1-dependent pathway, and facilitating tumor expansion.

Cancer cells that are able to evade immune surveillance require stimulation by cytokines and growth factors derived from numerous cell sources in order to proliferate. Aside from the factors secreted by HSCs and LSECs, tumor cells are stimulated by $\mathrm{KCs}$ present in the liver sinusoids, which are known to promote tumor progression at multiple levels, including promoting adhesion, proliferation, immune tolerance and angiogenesis (40). Ligand binding to ICAM-1 activates KC secretion of IL-6 (41), which induces cell proliferation via the STAT3 pathway, and stimulates the degradation of surrounding ECM via increased secretion of matrix metalloproteinases (MMPs) (101). IL-6 has been also implicated in the differentiation of human monocytes to M2 macrophages (102), a mechanism that probably also occurs in liver and is related to tumor progression (103). Interestingly, the infiltration of macrophages was reduced in ICAM-1 knockout mice in a model of renal injury (104), which is consistent with another report linking ICAM-1 blockage with a reduction in macrophage infiltration in precancerous pancreatic lesions (105). It is tempting to speculate that the same phenomenon may occur in the liver, as macrophages express LFA-1 and MUC-1, which are well known ICAM-1 ligands, suggesting that ICAM-1 is a key mediator in the recruitment of tumor-promoting macrophages. In fact, ICAM-1 silencing significantly reduced F4/80-expressing cells within the liver metastasis foci of C26 tumor-bearing mice (56).

The angiogenic response: Calling accomplices. An additional key and rate-limiting step in the metastatic process of a wide variety of tumors is the development of new blood vessels in order to fulfill the metabolic requirements of the tumor mass. The process of de novo formation of tumor blood vessels, tumor angiogenesis, determines the viability and survival of tumor cells and, thus, the establishment and development of the disease. The generation of new capillaries is favored by the stimuli present in the tumor microenvironment, produced by the tumor itself, by liver cells and by recruited immune cells. LSECs are required to form new capillary structures, with contractile HSCs necessary to provide support for new vessel formation (97). Our group revealed that LFA-1-expressing C26 cells interact with LSECs via ICAM-1, and that this interaction was the initial angiogenic stimulus during the early stage of liver metastasis, promoting tumor release of vascular endothelial growth factor (VEGF) (27,32), and driving the directional migration of LSECs and HSC recruitment to tumor areas (97). To further amplify this reaction, tumor cells, tumor-associated macrophages (TAMs) and myeloid-derived suppressor cells (MDSCs) contribute to VEGF secretion in response to their stimulation by TNF- $\alpha$, IL- 6 and NO, which are released through ICAM-1-mediated signaling pathways in KCs and LSECs $(106,107)$. Wang and Doerschuk reported that upon ICAM-1 ligand binding, pulmonary endothelial cells undergo cytoskeletal reorganization (108), a process required for the formation of new vessels, which may also occur in the liver when interacting with ICAM-1 ligands expressed on tumor cells. Recruitment of HSCs is crucial for the support of new vessels. HSCs respond to VEGF produced by tumor-activated cells and migrate to the sites of angiogenesis (109). The p38 mitogen-activated protein kinase (MAPK) pathway has been recently proposed as a mediator of endothelial cell activation, cytoskeletal reorganization and migration of HSCs $(110,111)$. Interestingly, ICAM-1 activation has been shown to stimulate the p38 MAPK pathway in endothelial cells, astrocytes and renal fibroblasts (112-114). Moreover, this MAPK pathway is also activated by platelet-derived growth factor, the most potent mitogen for HSCs (115), suggesting that ligand binding to ICAM-1 is a possible mitogenic stimulus responsible for the proliferative phenotype of these myofibroblast-like cells, expanding the number of activated cells ready to support angiogenesis (Fig. 2).

In addition to the angiogenic response, a desmoplastic reaction is observed during metastatic invasion of the liver. HSCs are the main producers of ECM proteins in the liver. In fact, the progression of CRC is associated with the desmoplastic reaction (116). In fibroblasts isolated from human CRC samples, ICAM-1 was increased in tumor-associated fibroblasts and was associated with an increase in desmoplasia in tissue sections (117). In liver metastasis induced by sICAM-1-activated C26 cells, an increase in collagen deposition is correlated with the infiltration of HSCs and with CD31-positive cells, indicating that the desmoplastic reaction is coupled to the angiogenic response and mediated by ICAM-1 (Arteta et al, Abstract in 12th International Symposium on Cells of the hepatic sinusoids, Bilbao, spain, 2004).

Immune cell recruitment: Allowing the arrival of infiltrating enemies. As mentioned previously, recruited immune populations infiltrate the liver via ICAM-1 adhesion. In order to efficiently fight against infiltrating tumor cells, either resident or recruited lymphocyte populations are required in order to mount an adequate immune response against the invading tumor. $\mathrm{CD}^{+} \mathrm{T}$ lymphocytes are able to control metastatic growth upon activation; however, when depleted, disease progression continues $(118,119)$. On the other hand, CD4 ${ }^{+}$ T lymphocytes also contribute to immune defense against cancer development (120). However, among the immune cell populations recruited from the circulation, MDSCs are able to suppress $\mathrm{T}$ cell cytotoxic activity towards the tumor and promote immune suppression (106). In this context, TAMs and MDSCs also contribute to tumor progression by decreasing anti-tumor immunity and generating a pro-tumor microenvironment (106,121-124). In fact, macrophages induce apoptosis of peripheral T cells following binding of LFA-1 to ICAM-1 (125), therefore promoting tumor survival through depletion of cytotoxic cells. Increased numbers of these immune cell populations, known to express LFA-1 receptor and MUC-1, are associated with the metastatic development of different cancers. In fact, the involvement of ICAM-1 in the 
adhesion of myeloid cell populations in vitro was reported by Makgoba et al in 1988 (126). Subsequently, other studies have reported the direct role of ICAM-1 in the adhesion of myeloid cells to dermal fibroblasts (127). Other authors have linked the increased expression of ICAM-1 with the infiltration of TAMs in renal carcinoma (128). Furthermore, this ICAM-1-mediated infiltration was also augmented in pancreatic cancer and correlated with the formation of precancerous lesions (105). Moreover, ICAM-1 silencing in C26 tumor-bearing mice resulted in decreased myeloid infiltration in metastatic liver lesions (56). Consequently, the expression of ICAM-1 may represent a potential therapeutic target for treating immune suppression during liver metastasis.

Recently, interest in the role of neutrophils as promoters of metastatic progression has grown. In ductal pancreatic adenocarcinoma, a significant association between the neutrophil count and the overall survival of the patients was reported, along with a correlation between the presence of neutrophils and distant metastasis after surgery (129). A relationship between neutrophils and metastasis formation in several organs, including the liver, has also been observed in other primary tumor types, such as breast carcinoma and CRC $(130,131)$. However, the role of host ICAM-1 in this process is unknown. In chronic inflammation of the liver, an increase of ICAM-1 on LSECs is known to mediate their interaction with neutrophils through CD18 integrin, favoring transmigration and adhesion to hepatocytes (132). Thus, it is feasible to consider that the same mechanism may be involved in neutrophil recruitment after CRC cell invasion in the liver. In fact, it has been shown that neutrophils bind to the liver endothelium through ligation to ICAM-1, facilitating the adhesion of melanoma cells (133), a process that may also be mediated by KCs. The fact that ICAM-1 expression on LSECs induced by KC-derived TNF- $\alpha$ leads to neutrophil adhesion and migration into the hepatic sinusoids after liver injury (134) supports the previous observation. Additionally, neutrophils take an active role during angiogenesis by secreting high levels of MMP-9. In fact, in gastric cancer, neutrophils promote the immune evasion of tumor cells and also the formation of new vessels (135).

Soluble ICAM-1: A moving threat. The influence of ICAM-1 goes beyond its membrane-bound form. In 1991, a soluble form of ICAM-1 was detected in human serum (136). Subsequently, chromatography and antibody detection revealed the significance of this finding, demonstrating the ability of this soluble isoform to interact with the membrane-bound ligands, such as LFA-1, as sICAM-1 contains a large fragment of the extracellular region of ICAM-1 (137). The serum level of circulating sICAM-1 is considered as a biomarker for several vascular inflammatory diseases, as well as in several different cancer types, such as breast, lung and colon cancers, and for lung and liver metastases (138-140). Interestingly, serum levels of sICAM-1 in patients with liver metastasis were found to be the highest among patients with different organ metastases (140). In the liver sICAM-1 is secreted from diverse cell types, including mononuclear cells, endothelial cells, fibroblasts, KCs and hepatocytes. During liver metastasis, several pathways are mediated by this soluble factor. Tumor-activated LSECs secrete sICAM-1 in response to various inflammatory stimuli, such as IL-1 $\beta$ (27), and thereby further enhance the metastatic capacity of tumor cells (40). It is tempting to hypothesize that sICAM-1 may promote the expression of other adhesion molecules in LSECs, as reported in human micro-vascular lung endothelial cells using different cancer cell lines (141). Among the responses to sICAM-1, proliferation of tumor and stromal cells has been observed in other cancer models (142). In fact, COX-2 was demonstrated to be activated in tumor cells upon sICAM-1 binding with LFA-1, resulting in an increase in the production of prostaglandin E2 (PGE2); PGE2 is a potent inflammatory, pro-immune tolerance and pro-angiogenic mediator, which stimulates the secretion of IL-1 $\beta$ by LSECs in an autocrine and paracrine loop (27), and ultimately increases the expression of membrane-bound ICAM-1 and vascular permeability, as mentioned previously (Fig. 2). Both IL-1 $\beta$ and PGE2 act as chemoattractants for MDSCs (143). Therefore, tumor cell binding to sICAM-1 results in an amplifying strategy for the recruitment of immune populations to develop an immune tolerant microenvironment in the liver. This immune tolerant status is further supported by the fact that sICAM-1 can interfere in the anti-tumor response, by inhibiting the interaction between patrolling $\mathrm{T}$ cells and cancer cells, and abrogating anti-tumor activity in natural killer cells $(144,145)$. Consequently, immune recognition leading to tumor cell clearance may be reduced by the increased level of circulating sICAM-1. Therefore, sICAM-1 acts as a messenger of the membrane-bound ICAM-1 activation signals, moving across the tumor vasculature to further enhance the pro-metastatic response of the liver.

\section{Conclusions}

ICAM-1 has been unequivocally shown to have a key role during tumor progression and metastasis formation in different organs. ICAM-1 is involved in the rearrangement of the actin cytoskeleton, the activation of pro-inflammatory cascades, and the mediation of multiple signaling pathways that regulate metastasis, such as tumor cell adhesion and transmigration, immune escape, desmoplasia and angiogenesis. However, even though the mechanisms by which the malignant cell expression of ICAM-1 mediates the aggressiveness of tumor cells have received much research attention, and are better understood, the role of ICAM-1 expression on host organ cells has not been a major focus of investigation. The expression of ICAM-1 on the surface of various resident liver cells with roles in different events during tumor invasion and colonization indicates that ICAM-1 could be a potential target for complementary and personalized therapies, as well as a powerful diagnostic and prognostic marker. As shown in this review, ICAM-1 acts as a transducer molecule, able to initiate a strong inflammatory response, which in turn amplifies the reactions, leading to increased adhesion, extravasation and tumor foci formation. Moreover, the production of immune cell chemoattractants promotes the recruitment of immunoregulatory cell populations that reduce the immune surveillance of the tumor. Additionally, the activation of pro-angiogenic and pro-desmoplastic stromal cells though ICAM-1-mediated signaling pathways triggers the development of a pro-tumor stroma and new vessel development, which further favor the growth and ultimate colonization of the liver. 
Thus, tissue ICAM-1 expression may reflect the growth and metastatic status of multiple cancer types, and may be a factor to predict cancer metastasis to the liver. ICAM-1 may be involved in various stages, from the very initial stages of inflammation, tumor and leukocyte adhesion to sinusoidal endothelial cells, and the evasion of immune destruction, to the very late stages of directional migration, differentiation and colonization. In light of the present knowledge, host ICAM-1 interactions with ligands on tumor and host cells during the different steps of metastatic progression remain an attractive target for the development of anti-cancer strategies. This will open new possibilities for treatments based on ICAM-1 as a therapeutic target. However, further investigations into its biological effects and the underlying mechanisms are required in order to develop effective therapies that can block interactions mediated by ICAM-1 without interfering with the normal functions of the organism.

\section{Acknowledgements}

The present study was financially supported in part by a postdoctoral fellowship from the University of the Basque Country to A.B. and by funds from the Basque Government-Saiotek to B.A.

\section{References}

1. Malietzis G, Lee GH, Bernardo D, Blakemore AI, Knight SC, Moorghen M, Al-Hassi HO and Jenkins JT: The prognostic significance and relationship with body composition of CCR7-positive cells in colorectal cancer. J Surg Oncol 112: 86-92, 2015.

2. Altendorf-Hofmann A and Scheele J: A critical review of the major indicators of prognosis after resection of hepatic metastases from colorectal carcinoma. Surg Oncol Clin N Am 12: 165-192, 2003.

3. Vidal-Vanaclocha F: The prometastatic microenvironment of the liver. Cancer Microenviron 1: 113-129, 2008.

4. Scheele J, Stangl R and Altendorf-Hofmann A: Hepatic metastases from colorectal carcinoma: Impact of surgical resection on the natural history. Br J Surg 77: 1241-1246, 1990.

5. Donadon M, Ribero D, Morris-Stiff G, Abdalla EK and Vauthey JN: New paradigm in the management of liver-only metastases from colorectal cancer. Gastrointest Cancer Res 1: 20-27, 2007.

6. Haier J, Korb T, Hotz B, Spiegel HU and Senninger N: An intravital model to monto steps of metastatic tumor cell adhesion within the hepatic microcirculation. J Gastrointest Surg 7: 507-514, 2003.

7. Van den Eyden GG, Majeed AW, Illemann M, Vermeulen PB, Bird NC, Høyer-Hansen G, Eefsen RL, Reynolds AR and Brodt P: The multifaceted role of the microenvironment in liver metastasis: Biology and clinical implications. Cancer Res 73 2031-2043, 2013

8. Rose DM, Essner R, Hughes TM, Tang PC, Bilchik A, Wanek LA, Thompson JF and Morton DL: Surgical resection for metastatic melanoma to the liver: The John Wayne cancer institute and sydney melanoma unit experience. Arch Surg 136: 950-955, 2001.

9. Eichbaum MH, Kaltwasser M, Bruckner T, de Rossi TM, Schneeweiss A and Sohn C: Prognostic factors for patients with liver metastases from breast cancer. Breast Cancer Res Treat 96: 53-62, 2006.

10. Yang TX, Chua TC and Morris DL: Radioembolization and chemoembolization for unresectable neuroendocrine liver metastases-a systematic review. Surg Oncol 21: 299-308, 2012.

11. Klein CA: Cancer. The metastasis cascade. Science 321: 1785-1787, 2008.

12. Stasinopoulos I,Penet MF, Krishnamachary B and Bhujwalla ZM Molecular and functional imaging of invasion and metastasis: Windows into the metastatic cascade. Cancer Biomark 7: 173-188, 2010.

13. Paschos KA, Canovas D and Bird NC: The role of cell adhesion molecules in the progression of colorectal cancer and the development of liver metastasis. Cell Signal 21: 665-674, 2009.
14. Kawaguchi T: Organ preference of cancer metastasis and metastasis-related cell adhesion molecules including carbohydrates. Cardiovasc Hematol Disord Drug Targets 15: 164-186, 2016.

15. Francavilla $\mathrm{C}$, Maddaluno $\mathrm{L}$ and Cavallaro $\mathrm{U}$ : The functional role of cell adhesion molecules in tumor angiogenesis. Semin Cancer Biol 19: 298-309, 2009.

16. Mueller MM and Fusenig NE: Friends or foes-bipolar effects of the tumour stroma in cancer. Nat Rev Cancer 4: 839-849, 2004.

17. Fridman WH,Remark R,GocJ,GiraldoNA,BechtE,Hammond SA, Damotte D, Dieu-Nosjean MC and Sautès-Fridman C: The immune microenvironment: A major player in human cancers. Int Arch Allergy Immunol 164: 13-26, 2014.

18. McDonald PC, Chafe SC and Dedhar S: Overcoming hypoxia-mediated tumor progression: Combinatorial approaches targeting $\mathrm{pH}$ Regulation, angiogenesis and immune dysfunction. Front Cell Dev Biol 4: 27, 2016.

19. Langley RR and Fidler IJ: The seed and soil hypothesis revisited-the role of tumor-stroma interactions in metastasis to different organs. Int J Cancer 128: 2527-2535, 2011.

20. Kobayashi H, Boelte KC and Lin PC: Endothelial cell adhesion molecules and cancer progression. Curr Med Chem 14: 377-386, 2007.

21. Arabzadeh A, Chan C, Nouvion AL, Breton V, Benlolo S, DeMarte L, Turbide C, Brodt P, Ferri L and Beauchemin N: Host-related carcinoembryonic antigen cell adhesion molecule 1 promotes metastasis of colorectal cancer. Oncogene 32: 849-860, 2013.

22. Khatib AM, Auguste P, Fallavollita L, Wang N, Samani A, Kontogiannea M, Meterissian S and Brodt P: Characterization of the host proinflammatory response to tumor cells during the initial stages of liver metastasis. Am J Pathol 167: 749-759, 2005.

23. Rahn JJ, Chow JW, Horne GJ, Mah BK, Emerman JT, Hoffman P and Hugh JC: MUCl mediates transendothelial migration in vitro by ligating endothelial cell ICAM-1. Clin Exp Metastasis 22: 475-483, 2005.

24. Laurent VM, Duperray A, Sundar Rajan V and Verdier C: Atomic force microscopy reveals a role for endothelial cell ICAM-1 expression in bladder cancer cell adherence. PLoS One 9: e98034, 2014.

25. Palange AL, Di Mascolo D, Carallo C, Gnasso A and Decuzzi P: Lipid-polymer nanoparticles encapsulating curcumin for modulating the vascular deposition of breast cancer cells. Nanomedicine 10: 991-1002, 2014.

26. Clayton A, Evans RA, Pettit E, Hallett M, Williams JD and Steadman R: Cellular activation through the ligation of intercellular adhesion molecule-1. J Cell Sci 111: 443-453, 1998.

27. Arteta B, Lasuen N, Lopategi A, Sveinbjörnsson B, Smedsrød B and Vidal-Vanaclocha F: Colon carcinoma cell interaction with liver sinusoidal endothelium inhibits organ-specific antitumor immunity through interleukin-1-induced mannose receptor in mice. Hepatology 51: 2172-2182, 2010.

28. Delfortrie S, Pinte S, Mattot V, Samson C, Villain G, Caetano B, Lauridant-Philippin G, Baranzelli MC, Bonneterre J, Trottein F, et al: Egfl7 promotes tumor escape from immunity by repressing endothelial cell activation. Cancer Res 71: 7176-7186, 2011.

29. Ghislin S, Obino D, Middendorp S, Boggetto N, Alcaide-Loridan C and Deshayes F: LFA-1 and ICAM-1 expression induced during melanoma-endothelial cell co-culture favors the transendothelial migration of melanoma cell lines in vitro. BMC Cancer 12: 455, 2012.

30. Wang HT, Lee HI, Guo JH, Chen SH, Liao ZK, Huang KW, Torng PL and Hwang LH: Calreticulin promotes tumor lymphocyte infiltration and enhances the antitumor effects of immunotherapy by up-regulating the endothelial expression of adhesion molecules. Int J Cancer 130: 2892-2902, 2012.

31. Akeichi T, Mocevicius P, Deduchovas O, Salnikova O, Castro-Santa E, Büchler MW, Schmidt J and Ryschich E: $\alpha \mathrm{L} \beta 2$ integrin is indispensable for $\mathrm{CD}^{+} \mathrm{T}$-cell recruitment in experimental pancreatic and hepatocellular cancer. Int J Cancer 130 : 2067-2076, 2012.

32. Valcárcel M, Arteta B, Jaureguibeitia A, Lopategi A, Martínez I, Mendoza L, Muruzabal FJ, Salado C and Vidal-Vanaclocha F: Three-dimensional growth as multicellular spheroid activates the proangiogenic phenotype of colorectal carcinoma cells via LFA-1-dependent VEGF: Implications on hepatic micrometastasis. J Transl Med 6: 57, 2008.

33. Yin Z, Jiang G, Fung JJ, Lu L and Qian S: ICAM-1 expressed on hepatic stellate cells plays an important role in immune regulation. Microsurgery 27: 328-332, 2007. 
34. Bruns T, Zimmermann HW, Pachnio A, Li KK, Trivedi PJ, Reynolds G, Hubscher S, Stamataki Z, Badenhorst PW, Weston CJ, et al: CMV infection of human sinusoidal endothelium regulates hepatic $\mathrm{T}$ cell recruitment and activation J Hepatol 63: 38-49, 2015.

35. van Den Engel NK, Heidenthal E, Vinke A, Kolb $\mathrm{H}$ and Martin S: Circulating forms of intercellular adhesion molecule (ICAM)-1 in mice lacking membranous ICAM-1. Blood 95: $1350-1355,2000$

36. Pluskota E and D'Souza SE: Fibrinogen interactions with ICAM-1 (CD54) regulate endothelial cell survival. Eur J Biochem 267: 4693-4704, 2000.

37. Shen Q, Rahn JJ, Zhang J, Gunasekera N, Sun X, Shaw AR, Hendzel MJ,Hoffman P, Bernier A and Hugh JC: MUC1 initiates Src-CrkL-Rac1/Cdc42-mediated actin cytoskeletal protrusive motility after ligating intercellular adhesion molecule-1. Mol Cancer Res 6: 555-567, 2008.

38. Gulubova MV: Expression of cell adhesion molecules and their beta1 and beta 2 integrin ligands in human liver peliosis. Pathol Res Pract 201: 503-511, 2005

39. Oudar O, Moreau A, Feldmann G and Scoazec JY: Expression and regulation of intercellular adhesion molecule-1 (ICAM-1) in organotypic cultures of rat liver tissue. J Hepatol 29: 901-909, 1998.

40. Gangopadhyay A, Lazure DA and Thomas P: Adhesion of colorectal carcinoma cells to the endothelium is mediated by cytokines from CEA stimulated Kupffer cells. Clin Exp Metastasis 16: 703-712, 1998.

41. Yang L, Froio RM, Sciuto TE, Dvorak AM, Alon R and Luscinskas FW: ICAM-1 regulates neutrophil adhesion and transcellular migration of TNF- $\alpha$-activated vascular endothelium under flow. Blood 106: 584-592, 2005.

42. Lalor PF, Shields P, Grant A and Adams DH: Recruitment of lymphocytes to the human liver. Immunol Cell Biol 80: 52-64, 2002.

43. Lawson C, Ainsworth M, Yacoub $M$ and Rose M: Ligation of ICAM-1 on endothelial cells leads to expression of VCAM-1 via a nuclear factor-kappaB-independent mechanism. J Immunol 162: 2990-2996, 1999.

44. Selzner N, Selzner M, Odermatt B, Tian Y, Van Rooijen N and Clavien PA: ICAM-1 triggers liver regeneration through leukocyte recruitment and Kupffer cell-dependent release of TNF-alpha/IL-6 in mice. Gastroenterology 124: 692-700, 2003.

45. Witkowska AM and Borawska MH: Soluble intercellular adhesion molecule-1 (sICAM-1): An overview. Eur Cytokine Netw 15: 91-98, 2004.

46. Sprenger A, Schardt C, Rotsch M, Zehrer M, Wolf M, Havemann K and Heymanns J: Soluble intercellular adhesion molecule-1 in patients with lung cancer and benign lung diseases. J Cancer Res Clin Oncol 123: 632-638, 1997.

47. Maruo Y, Gochi A, Kaihara A, Shimamura H, Yamada T, Tanaka N and Orita K: ICAM-1 expression and the soluble ICAM-1 level for evaluating the metastatic potential of gastric cancer. Int J Cancer 100: 486-490, 2002.

48. Christiansen I, Gidlof C, Kälkner KM, Hagberg H, Bennmarker $\mathrm{H}$ and Tötterman T: Elevated serum levels of soluble ICAM-1 in non-Hodgkin's lymphomas correlate with tumour burden, disease activity and other prognostic markers. Br J Haematol 92: 639-646, 1996.

49. Zhu XW and Gong JP: Expression and role of icam-1 in the occurrence and development of hepatocellular carcinoma Asian Pac J Cancer Prev 14: 1579-1583, 2013.

50. Kotteas EA, Gkiozos I, Tsagkouli S, Bastas A, Ntanos I, Saif MW and Syrigos KN: Soluble ICAM-1 levels in small-cell lung cancer: Prognostic value for survival and predictive significance for response during chemotherapy. Med Oncol 30: 662, 2013.

51. Gassmann P, Kang ML, Mees ST and Haier J: In vivo tumor cell adhesion in the pulmonary microvasculature is exclusively mediated by tumor cell-endothelial cell interaction. BMC Cancer 10: 177, 2010.

52. Zhang P, Goodrich C, Fu C and Dong C: Melanoma upregulates ICAM-1 expression on endothelial cells through engagement of tumor CD44 with endothelial E-selectin and activation of a PKC $\alpha$-p38-SP-1 pathway. FASEB J 28: 4591-4609, 2014.

53. Sipos E, Chen L, András IE, Wrobel J, Zhang B, Pu H, Park M, Eum SY and Toborek M: Proinflammatory adhesion molecules facilitate polychlorinated biphenyl-mediated enhancement of brain metastasis formation. Toxicol Sci 126: 362-371, 2012.
54. Gong L, Mi HJ, Zhu H,Zhou X and Yang H: P-selectin-mediated platelet activation promotes adhesion of non-small cell lung carcinoma cells on vascular endothelial cells under flow. Mol Med Rep 5: 935-942, 2012

55. Park JS, Kim KM, Kim MH, Chang HJ, Baek MK, Kim SM and Jung YD: Resveratrol inhibits tumor cell adhesion to endothelial cells by blocking ICAM-1 expression. Anticancer Res 29: 355-362, 2009.

56. Benedicto A, Marquez J, Olaso E and Arteta B: LFA-1/ICAM-1 interaction switches on an orchestrated prometastatic microenvironmental shift during experimental liver metastasis of colon C26 cancer cells. abstract. Cancer Res 75: B10-B10, 2015.

57. Eaton KV, Yang HL, Giachelli CM and Scatena M: Engineering macrophages to control the inflammatory response and angiogenesis. Exp Cell Res 339: 300-309, 2015.

58. Steinhoff G, Behrend M, Schrader B, Duijvestijn AM and Wonigeit K: Expression patterns of leukocyte adhesion ligand molecules on human liver endothelia. Lack of ELAM-1 and CD62 inducibility on sinusoidal endothelia and distinct distribution of VCAM-1, ICAM-1, ICAM-2 and LFA-3. Am J Pathol 142: 481-488, 1993.

59. Kong J, Kong L, Kong J, Ke S, Gao J, Ding X, Zheng L, Sun $\mathrm{H}$ and Sun W: After insufficient radiofrequency ablation, tumor-associated endothelial cells exhibit enhanced angiogenesis and promote invasiveness of residual hepatocellular carcinoma. J Transl Med 10: 230, 2012.

60. Lee WY and Kubes P: Leukocyte adhesion in the liver: Distinct adhesion paradigm from other organs. J Hepatol 48: 504-512, 2008.

61. Salas A, Shimaoka M, Phan U, Kim M and Springer TA: Transition from rolling to firm adhesion can be mimicked by extension of integrin alphaLbeta2 in an intermediate affinity state. J Biol Chem 281: 10876-10882, 2006.

62. Roosien FF, de Kuiper PE, de Rijk D and Roos E: Invasive and metastatic capacity of revertants of LFA-1-deficient mutant T-cell hybridomas. Cancer Res 50: 3509-3513, 1990.

63. Tatsumi T, Shimazaki C, Goto H, Araki S, Sudo Y, Yamagata N, Ashihara E, Inaba T, Fujita $\mathrm{N}$ and Nakagawa M: Expression of adhesion molecules on myeloma cells. Jpn J Cancer Res 87: 837-842, 1996

64. Gulubova MV: Expression of cell adhesion molecules, their ligands and tumour necrosis factor alpha in the liver of patients with metastatic gastrointestinal carcinomas. Histochem J 34: $67-77,2002$.

65. Soto MS, Serres S, Anthony DC and Sibson NR: Functional role of endothelial adhesion molecules in the early stages of brain metastasis. Neuro-Oncol 16: 540-551, 2014.

66. Horm TM and Schroeder JA: MUC1 and metastatic cancer: Expression, function and therapeutic targeting. Cell Adh Migr 7: 187-198, 2013.

67. Williams K, Motiani K, Giridhar PV and Kasper S: CD44 integrates signaling in normal stem cell, cancer stem cell and (pre)metastatic niches. Exp Biol Med (Maywood) 238: 324-338, 2013.

68. Olsson E, Honeth G, Bendahl PO, Saal LH, Gruvberger-Saal S, Ringnér M, Vallon-Christersson J, Jönsson G, Holm K, Lövgren $\mathrm{K}$, et al: CD44 isoforms are heterogeneously expressed in breast cancer and correlate with tumor subtypes and cancer stem cell markers. BMC Cancer 11: 418, 2011.

69. Dalerba P, Dylla SJ, Park IK, Liu R, Wang X, Cho RW, Hoey T, Gurney A, Huang EH, Simeone DM, et al: Phenotypic characterization of human colorectal cancer stem cells. Proc Natl Acad Sci USA 104: 10158-10163, 2007.

70. Li C, Heidt DG, Dalerba P, Burant CF, Zhang L, Adsay V, Wicha M, Clarke MF and Simeone DM: Identification of pancreatic cancer stem cells. Cancer Res 67: 1030-1037, 2007.

71. Ohtani H: Pathophysiologic significance of host reactions in human cancer tissue: Desmoplasia and tumor immunity. Tohoku J Exp Med 187: 193-202, 1999.

72. Essani NA, McGuire GM, Manning AM and Jaeschke H: Differential induction of mRNA for ICAM-1 and selectins in hepatocytes, Kupffer cells and endothelial cells during endotoxemia. Biochem Biophys Res Commun 211: 74-82, 1995.

73. Usami Y, Ishida K, Sato S, Kishino M, Kiryu M, Ogawa Y, Okura M, Fukuda Y and Toyosawa S: Intercellular adhesion molecule-1 (ICAM-1) expression correlates with oral cancer progression and induces macrophage/cancer cell adhesion. Int J Cancer 133: 568-578, 2013 
74. Ohira H , Ueno T, Shakado S, Sakamoto M, Torimura T, Inuzuka S, Sata M and Tanikawa K: Cultured rat hepatic sinusoidal endothelial cells express intercellular adhesion molecule-1 (ICAM-1) by tumor necrosis factor-alpha or interleukin-1 alpha stimulation. J Hepatol 20: 729-734, 1994.

75. Tacconi C, Correale C, Gandelli A, Spinelli A, Dejana E, D'Alessio $S$ and Danese S: Vascular endothelial growth factor $C$ disrupts the endothelial lymphatic barrier to promote colorectal cancer invasion. Gastroenterology 148: 1438-1451, 2015.

76. Weber MR, Zuka M, Lorger M, Tschan M, Torbett BE, Zijlstra A, Quigley JP, Staflin K, Eliceiri BP, Krueger JS, et al: Activated tumor cell integrin $\alpha v \beta 3$ cooperates with platelets to promote extravasation and metastasis from the blood stream. Thromb Res 140 (Suppl 1): S27-S36, 2016.

77. Locard-Paulet M, Lim L, Veluscek G, McMahon K, Sinclair J, van Weverwijk A, Worboys JD, Yuan Y, Isacke CM and Jørgensen C: Phosphoproteomic analysis of interacting tumor and endothelial cells identifies regulatory mechanisms of transendothelial migration. Sci Signal 9: ra15, 2016.

78. Skau CT, Fischer RS, Gurel P, Thiam HR, Tubbs A, Baird MA, Davidson MW, Piel M, Alushin GM, Nussenzweig A, et al: FMN2 makes perinuclear actin to protect nuclei during confined migration and promote metastasis. Cell 167 1571-1585, 2016.

79. Freeman SA, McLeod SJ, Dukowski J, Austin P, Lee CC, Millen-Martin B, Kubes P, McCafferty DM, Gold MR and Roskelley CD: Preventing the activation or cycling of the Rap1 GTPase alters adhesion and cytoskeletal dynamics and blocks metastatic melanoma cell extravasation into the lungs. Cancer Res 70: 4590-4601, 2010.

80. Sato T, Habtezion A, Beilhack A, Schulz S, Butcher E and Thorlacius H: Short-term homing assay reveals a critical role for lymphocyte function-associated antigen-1 in the hepatic recruitment of lymphocytes in graft-versus-host disease. J Hepatol 44 1132-1140, 2006.

81. Gorina R, Lyck R, Vestweber D and Engelhardt B: $\beta 2$ integrin-mediated crawling on endothelial ICAM-1 and ICAM-2 is a prerequisite for transcellular neutrophil diapedesis across the inflamed blood-brain barrier. J Immunol 192: 324-337, 2014.

82. Fu C, Tong C, Wang M, Gao Y, Zhang Y, Lü S, Liang S, Dong C and Long $\mathrm{M}$ : Determining beta2-integrin and intercellular adhesion molecule 1 binding kinetics in tumor cell adhesion to leukocytes and endothelial cells by a gas-driven micropipette assay. J Biol Chem 286: 34777-34787, 2011.

83. Haddad O, Chotard-Ghodsnia R, Verdier C and Duperray A Tumor cell/endothelial cell tight contact upregulates endothelia adhesion molecule expression mediated by NFkappaB: Differential role of the shear stress. Exp Cell Res 316: 615-626, 2010.

84. Wong J, Johnston B, Lee SS, Bullard DC, Smith CW, Beaudet AL and Kubes P: A minimal role for selectins in the recruitment of leukocytes into the inflamed liver microvasculature. J Clin Invest 99: 2782-2790, 1997.

85. Ronald JA, Ionescu CV, Rogers KA and Sandig M: Differential regulation of transendothelial migration of THP-1 cells by ICAM-1/LFA-1 and VCAM-1/VLA-4. J Leukoc Biol 70: 601-609, 2001.

86. Kubes P and Mehal WZ: Sterile inflammation in the liver. Gastroenterology 143: 1158-1172, 2012.

87. Ramadori G, Moriconi F, Malik I and Dudas J: Physiology and pathophysiology of liver inflammation, damage and repair. J Physiol Pharmacol 59 (Suppl 1): S107-S117, 2008

88. Coussens LM and Werb Z: Inflammation and cancer. Nature 420: 860-867, 2002.

89. Weis SM and Cheresh DA: Tumor angiogenesis: Molecular pathways and therapeutic targets. Nat Med 17: 1359-1370, 2011.

90. Whiteside TL: Immune suppression in cancer: Effects on immune cells, mechanisms and future therapeutic intervention. Semin Cancer Biol 16: 3-15, 2006.

91. Dvorak HF: Tumors: Wounds that do not heal. Similarities between tumor stroma generation and wound healing. N Engl J Med 315: 1650-1659, 1986

92. Lee UE and Friedman SL: Mechanisms of hepatic fibrogenesis. Best Pract Res Clin Gastroenterol 25: 195-206, 2011

93. Madar S, Goldstein I and Rotter V: 'Cancer associated fibroblasts'-more than meets the eye. Trends Mol Med 19: 447-453, 2013.

94. Hellerbrand Wang SC, Tsukamoto H, Brenner DA and Rippe RA: Expression of intracellular adhesion molecule 1 by activated hepatic stellate cells. Hepatology 24: 670-676, 1996.
95. Masamune A, Sakai Y, Kikuta K, Satoh M, Satoh A and Shimosegawa T: Activated rat pancreatic stellate cells express intercellular adhesion molecule-1 (ICAM-1) in vitro. Pancreas 25: 78-85, 2002.

96. Kang N, Gores GJ and Shah VH: Hepatic stellate cells: Partners in crime for liver metastases? Hepatology 54: 707-713, 2011.

97. Olaso E, Salado C, Egilegor E, Gutierrez V, Santisteban A and Sancho-Bru P, Friedman SL and Vidal-Vanaclocha F: Proangiogenic role of tumor-activated hepatic stellate cells in experimental melanoma metastasis. Hepatology 37: 674-685, 2003.

98. Muhanna N, Doron S, Wald O, Horani A, Eid A, Pappo O, Friedman SL and Safadi R: Activation of hepatic stellate cells after phagocytosis of lymphocytes: A novel pathway of fibrogenesis. Hepatology 48: 963-977, 2008.

99. Schildberg FA, Wojtalla A, Siegmund SV, Endl E, Diehl L, Abdullah Z, Kurts C and Knolle PA: Murine hepatic stellate cells veto $\mathrm{CD} 8 \mathrm{~T}$ cell activation by a CD54-dependent mechanism. Hepatology 54: 262-272, 2011.

100.Zhao W, Zhang L, Xu Y, Zhang Z, Ren G, Tang K, Kuang P, Zhao B, Yin Z and Wang X: Hepatic stellate cells promote tumor progression by enhancement of immunosuppressive cells in an orthotopic liver tumor mouse model. Lab Invest 94: 182-191, 2014

101. Fisher DT, Appenheimer MM and Evans SS: The two faces of IL-6 in the tumor microenvironment. Semin Immunol 26 38-47, 2014.

102. Roca H, Varsos ZS, Sud S, Craig MJ, Ying C and Pienta KJ: CCL2 and interleukin- 6 promote survival of human CD11b peripheral blood mononuclear cells and induce M2-type macrophage polarization. J Biol Chem 284: 34342-34354, 2009.

103. Galdiero MR, Bonavita E, Barajon I, Garlanda C, Mantovani A and Jaillon S: Tumor associated macrophages and neutrophils in cancer. Immunobiology 218: 1402-1410, 2013

104. Okada S, Shikata K, Matsuda M, Ogawa D, Usui H, Kido Y, Nagase R, Wada J, Shikata Y and Makino H: Intercellular adhesion molecule-1-deficient mice are resistant against renal injury after induction of diabetes. Diabetes 52: 2586-2593, 2003.

105. Liou GY, Döppler H, Necela B, Edenfield B, Zhang L, Dawson DW and Storz P: Mutant KRAS-induced expression of ICAM-1 in pancreatic acinar cells causes attraction of macrophages to expedite the formation of precancerous lesions. Cancer Discov 5: 52-63, 2015.

106. Lee BR, Chang SY, Hong EH, Kwon BE, Kim HM, Kim YJ, Lee J, Cho HJ, Cheon JH and Ko HJ: Elevated endoplasmic reticulum stress reinforced immunosuppression in the tumor microenvironment via myeloid-derived suppressor cells. Oncotarget 5: 12331-12345, 2014.

107. Sander LE, Sackett SD, Dierssen U, Beraza N, Linke RP, Müller M, Blander JM, Tacke F and Trautwein C: Hepatic acute-phase proteins control innate immune responses during infection by promoting myeloid-derived suppressor cell function. J Exp Med 207: 1453-1464, 2010.

108. Wang Q and Doerschuk CM: The p38 mitogen-activated protein kinase mediates cytoskeletal remodeling in pulmonary microvascular endothelial cells upon intracellular adhesion molecule-1 ligation. J Immunol 166: 6877-6884, 2001.

109. Novo E, Cannito S, Zamara E, Valfrè di Bonzo L, Caligiuri A, Cravanzola C, Compagnone A, Colombatto S, MarraF,Pinzani M and Parola M: Proangiogenic cytokines as hypoxia-dependent factors stimulating migration of human hepatic stellate cells. Am J Pathol 170: 1942-1953, 2007.

110. Li X, Wang X, Han C, Wang X, Xing G, Zhou L, Li G and Niu Y: Astragaloside IV suppresses collagen production of activated hepatic stellate cells via oxidative stress-mediated p38 MAPK pathway. Free Radic Biol Med 60: 168-176, 2013.

111. Cui X, Zhang X, Yin Q, Meng A, Su S, Jing X, Li H, Guan X, $\mathrm{Li} \mathrm{X}$, Liu S and Cheng M: F-actin cytoskeleton reorganization is associated with hepatic stellate cell activation. Mol Med Rep 9: 1641-1647, 2014

112. Wang Q, Pfeiffer GR II and Gaarde WA: Activation of SRC tyrosine kinases in response to ICAM-1 ligation in pulmonary microvascular endothelial cells. J Biol Chem 278: 47731-47743, 2003.

113. Lee SJ, Drabik K, Van Wagoner NJ, Lee S, Choi C, Dong Y and Benveniste EN: ICAM-1-induced expression of proinflammatory cytokines in astrocytes: Involvement of extracellular signal-regulated kinase and p38 mitogen-activated protein kinase pathways. J Immunol 165: 4658-4666, 2000. 
114. Blaber R, Stylianou E, Clayton A and Steadman R: Selective regulation of ICAM-1 and RANTES gene expression after ICAM-1 ligation on human renal fibroblasts. J Am Soc Nephrol 14: 116-127, 2003.

115. Borkham-Kamphorst E, van Roeyen CR, Ostendorf T, Floege J, Gressner AM and Weiskirchen R: Pro-fibrogenic potential of PDGF-D in liver fibrosis. J Hepatol 46: 1064-1074, 2007.

116. Vermeulen PB, Colpaert C, Salgado R, Royers R, Hellemans H, Van Den Heuvel E, Goovaerts G, Dirix LY and Van Marck E: Liver metastases from colorectal adenocarcinomas grow in three patterns with different angiogenesis and desmoplasia. J Pathol 195: 336-342, 2001.

117. Schellerer VS, Langheinrich M, Hohenberger W, Croner RS, Merkel S, Rau TT, Stürzl M and Naschberger E: Tumor-associated fibroblasts isolated from colorectal cancer tissues exhibit increased ICAM-1 expression and affinity for monocytes. Oncol Rep 31: 255-261, 2014.

118. Brackett CM, Kojouharov B, Veith J, Greene KF, Burdelya LG Gollnick SO, Abrams SI and Gudkov AV: Toll-like receptor-5 agonist, entolimod, suppresses metastasis and induces immunity by stimulating an NK-dendritic-CD $8^{+} \mathrm{T}$-cell axis. Proc Natl Acad Sci USA 113: E874-E883, 2016.

119. Poczobutt JM, Nguyen TT, Hanson D, Li H, Sippel TR, Weiser-Evans MC, Gijon M, Murphy RC and Nemenoff RA: Deletion of 5-Lipoxygenase in the tumor microenvironment promotes lung cancer progression and metastasis through regulating T cell recruitment. J Immunol 196: 891-901, 2016.

120.Zanetti M: Tapping CD4 T cells for cancer immunotherapy: The choice of personalized genomics. J Immunol 194: 2049-2056, 2015.

121. Umansky V, Blattner C, Gebhardt C and Utikal J: The Role of Myeloid-Derived Suppressor Cells (MDSC) in Cancer Progression. Vaccines (Basel) 4: E36, 2016.

122. Umansky V, Blattner C, Fleming V, Hu X, Gebhardt C, Altevogt $\mathrm{P}$ and Utikal J: Myeloid-derived suppressor cells and tumor escape from immune surveillance. Semin Immunopathol 39: 295-305, 2017

123. Baay M, Brouwer A, Pauwels P, Peeters M and Lardon F: Tumor cells and tumor-associated macrophages: Secreted proteins as potential targets for therapy. Clin Dev Immunol 2011: 565187, 2011.

124. Ostrand-Rosenberg S and Sinha P: Myeloid-derived suppressor cells: Linking inflammation and cancer. J Immunol 182: 4499-4506, 2009

125.Zen K, Masuda J and Ogata J: Monocyte-derived macrophages prime peripheral $\mathrm{T}$ cells to undergo apoptosis by cell-cell contact via ICAM-1/LFA-1-dependent mechanism. Immunobiology 195: 323-333, 1996

126. Makgoba MW, Sanders ME, Ginther Luce GE, Dustin ML, Springer TA, Clark EA, Mannoni P and Shaw S: ICAM-1 a ligand for LFA-1-dependent adhesion of B, T and myeloid cells. Nature 331: 86-88, 1988

127. Rabquer BJ, Hou Y, Del Galdo F, Haines GK III, Gerber ML, Jimenez SA, Seibold JR and Koch AE: The proadhesive phenotype of systemic sclerosis skin promotes myeloid cell adhesion via ICAM-1 and VCAM-1. Rheumatology (Oxford) 48: 734-740, 2009.

128.Hemmerlein B, Scherbening J, Kugler A and Radzun HJ: Expression of VCAM-1, ICAM-1, E- and P-selectin and tumour-associated macrophages in renal cell carcinoma. Histopathology 37: 78-83, 2000 .

129. Tao L, Zhang L, Peng Y, Tao M, Li L, Xiu D, Yuan C, Ma Z and Jiang B: Neutrophils assist the metastasis of circulating tumor cells in pancreatic ductal adenocarcinoma: A new hypothesis and a new predictor for distant metastasis. Medicine (Baltimore) 95: e4932, 2016.
130. Tabariès S, Ouellet V, Hsu BE, Annis MG, Rose AA, Meunier L, Carmona E, Tam CE, Mes-Masson AM and Siegel PM: Granulocytic immune infiltrates are essential for the efficient formation of breast cancer liver metastases. Breast Cancer Res 17: 45, 2015.

131. Hirai H, Fujishita T, Kurimoto K, Miyachi H, Kitano S, Inamoto S, Itatani Y, Saitou M, Maekawa T and Taketo MM: CCR1-mediated accumulation of myeloid cells in the liver microenvironment promoting mouse colon cancer. Clin Exp Metastasis 31: 977-989, 2014.

132. Ramaiah SK and Jaeschke H: Hepatic neutrophil infiltration in the pathogenesis of alcohol-induced liver injury. Toxicol Mech Methods 17: 431-440, 2007.

133. Slattery MJ, Liang S and Dong C: Distinct role of hydrodynamic shear in leukocyte-facilitated tumor cell extravasation. Am J Physiol Cell Physiol 288: C831-C839, 2005.

134. Sakamoto S, Okanoue T, Itoh Y, Nakagawa Y, Nakamura H, Morita A, Daimon Y, Sakamoto K, Yoshida N, Yoshikawa T and Kashima K: Involvement of Kupffer cells in the interaction between neutrophils and sinusoidal endothelial cells in rats. Shock 18: 152-157, 2002.

135. Li TJ, Jiang YM, Hu YF, Huang L, Yu J, Zhao LY, Deng HJ, Mou TY, Liu H, Yang Y, et al: Interleukin-17-producing neutrophils link inflammatory stimuli to disease progression by promoting angiogenesis in gastric cancer. Clin Cancer Res 23: $1575-1585,2016$.

136. Seth R, Raymond FD and Makgoba MW: Circulating ICAM-1 isoforms: Diagnostic prospects for inflammatory and immune disorders. Lancet 338: 83-84, 1991.

137. Rothlein R, Mainolfi EA, Czajkowski M and Marlin SD: A form of circulating ICAM-1 in human serum. J Immunol 147: 3788-3793, 1991.

138. Tesarova P, Kalousova M, Zima T, Suchanek M, Malikova I, Kvasnicka J, Duskova D, Tesar V, Vachek J, Krupickova-Kasalova Z and Malik J: Endotelial activation and flow-mediated vasodilation in young patients with breast cancer. Neoplasma 60: 690-697, 2013.

139. Guney N, Soydinc HO, Derin D, Tas F, Camlica H, Duranyildiz D, Yasasever V and Topuz E: Serum levels of intercellular adhesion molecule ICAM-1 and E-selectin in advanced stage non-small cell lung cancer. Med Oncol 25: 194-200, 2008.

140. Dymicka-Piekarska V, Guzinska-Ustymowicz K, Kuklinski A and Kemona H: Prognostic significance of adhesion molecules (sICAM-1, sVCAM-1) and VEGF in colorectal cancer patients. Thromb Res 129: e47-e50, 2012.

141. Chen C, Duckworth CA, Zhao Q, Pritchard DM, Rhodes JM and Yu LG: Increased circulation of galectin-3 in cancer induces secretion of metastasis-promoting cytokines from blood vascular endothelium. Clin Cancer Res 19: 1693-1704, 2013.

142. Takahara M, Nagato T, Komabayashi Y, Yoshino K, Ueda S, Kishibe K and Harabuchi Y: Soluble ICAM-1 secretion and its functional role as an autocrine growth factor in nasal NK/T cell lymphoma cells. Exp Hematol 41: 711-718, 2013.

143. Draghiciu O, Lubbers J, Nijman HW and Daemen T: Myeloid derived suppressor cells-An overview of combat strategies to increase immunotherapy efficacy. Oncoimmunology 4: e954829, 2015.

144. Becker JC, Dummer R, Hartmann AA, Burg G and Schmidt RE: Shedding of ICAM-1 from human melanoma cell lines induced by IFN-gamma and tumor necrosis factor-alpha. Functional consequences on cell-mediated cytotoxicity. J Immunol 147: 4398-4401, 1991.

145. Becker JC, Termeer C, Schmidt RE and Bröcker EB: Soluble intercellular adhesion molecule-1 inhibits MHC-restricted specific T cell/tumor interaction. J Immunol 151: 7224-7232, 1993. 